\title{
Quantum proteolysis by neutrophils: implications for pulmonary emphysema in $\alpha_{1}$-antitrypsin deficiency
}

\author{
Edward J. Campbell, Melody A. Campbell, Steve S. Boukedes, and Caroline A. Owen \\ Department of Internal Medicine, University of Utah Health Sciences Center, Salt Lake City, Utah 84132, USA \\ Address correspondence to: Edward J. Campbell, Department of Medicine, University of Utah Health Sciences Center, \\ 410 Chipeta Way, Room 108, Salt Lake City, Utah 84108, USA. \\ Phone: (801) 585-6192; Fax: (801) 585-3118; E-mail: edward.campbell@hsc.utah.edu.
}

Received for publication December 17, 1998, and accepted in revised form June 29, 1999.

Traditional enzyme kinetics provide a poor explanation for the increased risk of lung injury in $\alpha_{1^{-}}$ antitrypsin (AAT) deficiency. Millimolar concentrations of leukocyte elastase, when released from single azurophil granules of activated neutrophils, lead to evanescent quantum bursts of proteolytic activity before catalysis is quenched by pericellular inhibitors. Herein, we tested the possibility that quantum proteolytic events are abnormal in AAT deficiency. We incubated neutrophils on opsonized fluoresceinated fibronectin in serum from individuals with various AAT phenotypes, and then measured and modeled quantum proteolytic events. The mean areas of the events in serum from heterozygous individuals ( $\mathrm{Pi} \mathrm{MZ}$ and Pi SZ) were slightly, but significantly, larger than those in serum from normal patients (Pi M). In marked contrast, mean areas of events in serum from AAT-deficient individuals were 10 -fold larger than those in serum from normal patients. Diffusion modeling predicted that local elastase concentrations exceed AAT concentrations for less than 20 milliseconds and for more than 80 milliseconds in $\mathrm{Pi} \mathrm{M}$ and $\mathrm{Pi} Z$ individuals, respectively. Thus, quantum proteolytic events are abnormally large and prolonged in AAT deficiency, leading directly to an increased risk of tissue injury in the immediate vicinity of activated neutrophils. These results have potentially important implications for the pathogenesis and prevention of lung disease in AAT deficiency.

J. Clin. Invest. 104:337-344 (1999).

\section{Introduction}

Deficiency of $\alpha_{1}$-antitrypsin (AAT) is the most common, potentially lethal hereditary disease seen in Caucasians. AAT is a highly polymorphic serine proteinase inhibitor and is the major inhibitor of human leukocyte elastase (HLE) in plasma and in the lower respiratory tract. Individuals with AAT deficiency have a markedly increased risk of severe, early-onset pulmonary emphysema (1). HLE is contained at high concentrations within the azurophil granules of polymorphonuclear neutrophils (PMNs) (2) and within the peroxidase-positive granules of proinflammatory $(\mathrm{P})$ monocytes (3). It is thought that the increased risk of lung tissue injury in AAT-deficient individuals is due to inadequately controlled HLE activity that is released from activated inflammatory cells within the lower respiratory tract (4).

Individuals with $\mathrm{Pi} Z$ phenotype have plasma concentrations of AAT (5) that are $10-15 \%$ that of normal (mean is $\sim 32.8 \mu \mathrm{M}$ in Pi M individuals). However, heterozygotes and individuals with other common AAT variants (including Pi MS, Pi SS, and Pi MZ individuals) have intermediate plasma AAT concentrations $(\sim 15-30 \mu \mathrm{M})$ and have minimal, if any, increased risk of developing emphysema when compared with Pi M individuals (reviewed in ref. 6). More interestingly, $\mathrm{Pi}$ SZ heterozygotes with plasma AAT concentrations in the 8-19 $\mu \mathrm{M}$ range also have minimal excess risk of lung disease $(6,7)$.
Traditional enzyme kinetics have failed to provide a satisfactory explanation for the strikingly increased risk of lung tissue injury that occurs in $\mathrm{Pi} Z$ individuals, because (a) even in Pi $Z$ individuals, the mean AAT concentration in plasma $(\sim 5.3 \mu \mathrm{M})$ is more than 7 orders of magnitude greater than its $K_{\mathrm{I}}$ for HLE $(3.3 \times$ $\left.10^{-14} \mathrm{M}\right)(8)$; and (b) only subtle abnormalities in the function of the $Z$ variant have been described $(9,10)$. In this respect, it is noteworthy that recent studies from our laboratory (11) have demonstrated that evanescent, quantized bursts of HLE-mediated proteolytic activity are associated with the extracellular release of high concentrations of HLE from single azurophil granules of PMNs. Our diffusion-based theoretical model (2) predicts that obligate HLE catalytic activity persists until HLE diffuses away from the site of granule release and the molar ratio of enzyme to extracellular inhibitor decreases to less than 1:1.

Herein, we have tested the possibility that the quantum proteolytic events associated with PMN degranulation are markedly abnormal in $\mathrm{Pi} Z$ individuals and thereby directly contribute to the excess risk of lung tissue injury that is observed in AAT deficiency. To accomplish this, we directly measured and modeled quantum events occurring while PMNs were bathed in serum from donors having different AAT phenotypes. Our results confirmed that quantum proteolytic events can be expected to be greatly different, both in 

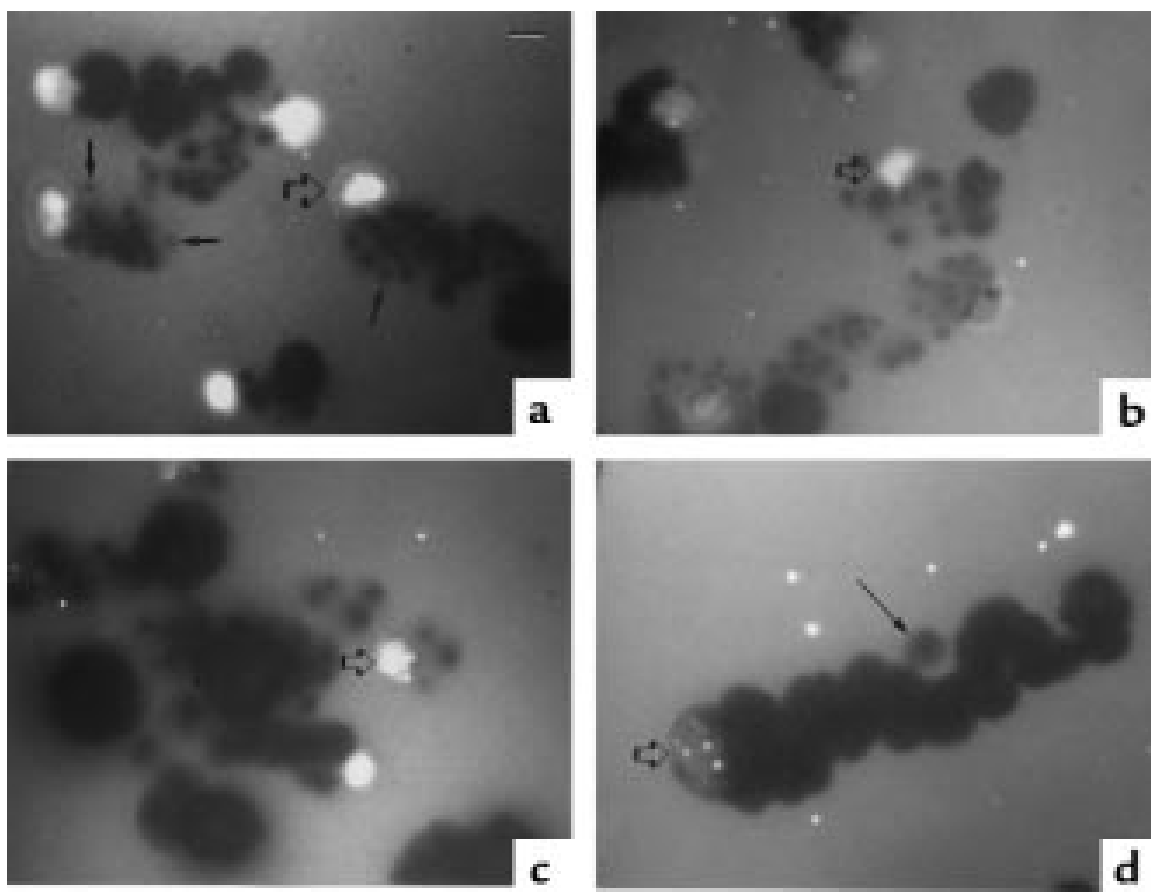

\section{Figure 1}

Quantum proteolytic events associated with PMNs bathed in serum from donors with various AAT phenotypes. PMNs were allowed to migrate for 30 minutes at $37^{\circ} \mathrm{C}$ on opsonized fluoresceinated fibronectin while bathed in serum from phenotypes with Pi M (a), Pi MZ (b), Pi SZ (c), and Pi Z (d). Cells were fixed and then examined by incident-light fluorescence microscopy. Note the discrete, rounded, dark areas associated with migrating cells, which are very localized areas of degraded fibronectin (short arrows in a identify selected areas of quantum proteolysis). Note also the coalescent areas of degradation that are related to release of 2 or more azurophil granules. PMNs remaining on the surface are fluorescent because of ingested fibronectin fragments (broad arrows). The quantum proteolytic events are visually similar in size in a-c. Note also that cell-associated proteolytic events are strikingly larger in cells bathed in Pi Z serum (long arrow in d) when compared with those bathed in serum from the other donors. Scale bar: $10 \mu \mathrm{m}$.

size and duration, in AAT deficiency when compared with normal individuals and heterozygotes. These data have implications for the pathogenesis and prevention of lung injury in AAT deficiency.

\section{Methods}

Reagents. Human plasma fibronectin, FMLP, LPS from Escherichia coli 0111:B4, TRITC, rabbit anti-human fibronectin IgG, $p$-phenylenediamine, paraformaldehyde, and glutaraldehyde were obtained from Sigma Chemical Co. (St. Louis, Missouri, USA). HBSS and 1 $M$ HEPES buffered solution were purchased from GIBCO BRL (Grand Island, New York, USA).

Serum. Serum was collected from individuals with varying AAT phenotypes (Pi M, Pi MS, Pi MZ, Pi SZ, and Pi $Z$ ), as determined by isoelectric focusing (12). AAT levels were determined by automated fluorescence immunoassay (13).

PMN isolation. Human PMNs were isolated from the peripheral blood of healthy volunteers using ficollHypaque centrifugation followed by dextran sedimentation (14). PMNs were resuspended at $4 \times 10^{6} / \mathrm{mL}$ in HBSS ( $\mathrm{pH} 7.4$ ) at $4^{\circ} \mathrm{C}$ and used promptly in the experiments detailed below.

Preparation of substrate surface. Human plasma fibronectin was conjugated to TRITC (11). Individual wells of LabTek glass chamber slides (Nalge Nunc
International, Naperville, Illinois, USA) were coated overnight at $4^{\circ} \mathrm{C}$, in the dark, with $20 \mu \mathrm{g}$ of fibronectinTRITC. The fibronectin-TRITC surfaces were washed 3 times with HBSS, opsonized with rabbit antifibronectin $\operatorname{IgG}$ for 1 hour at $37^{\circ} \mathrm{C}$, and washed 3 additional times to remove nonadsorbed proteins.

Quantitative imaging of quantum proteolytic events. HEPES (25 mM, pH 7.1) was added to serum from donors of varying AAT phenotypes to buffer the serum samples in ambient air. Aliquots $(150 \mu \mathrm{L})$ of the serum samples were then added to wells of the coated glass chamber slides, followed by the addition of freshly isolated PMNs $(20,000$ cells in $5 \mu \mathrm{L})$. This procedure resulted in only a minimal dilution of the serum in the final suspension, which was $94.4 \%$ serum.

The chamber slides were incubated in the dark for 30 minutes at $37^{\circ} \mathrm{C}$, the wells were washed twice with HBSS, and then the cells were fixed for 5 minutes with PBS containing 3\% (wt/vol) paraformaldehyde and $1 \%(\mathrm{vol} / \mathrm{vol})$ glutaraldehyde ( $\mathrm{pH}$ 7.4). The wells were washed twice in HBSS, the chambers were removed, and the slide was mounted in PBS containing 25\% (vol/vol) glycerol and $250 \mu \mathrm{g} / \mathrm{mL}$-phenylenediamine to reduce photobleaching. The slides were examined with phase-contrast and incident-light fluorescence microscopy (Leitz Dialux 20 with L2 filter set and Leitz NPL Fluotar $\times 50$, NA 1.00 objective; Leica GmbH, Wetzlar, Germany). Images of the 


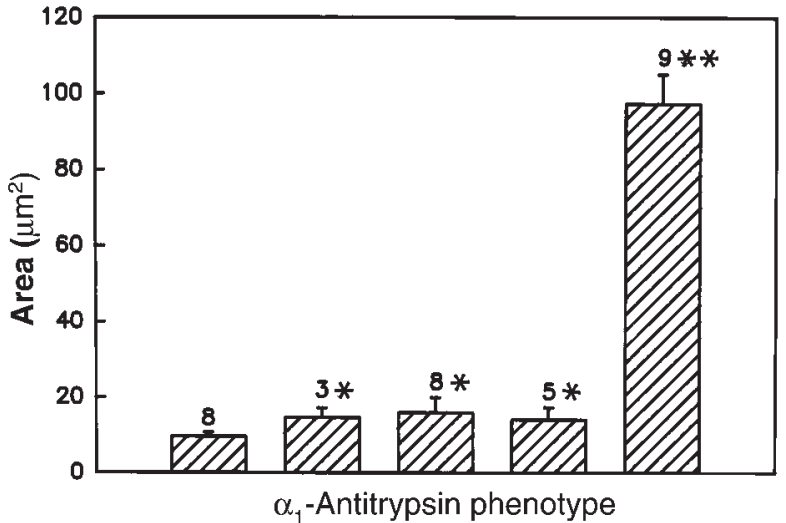

Figure 2

Size of PMN quantum proteolytic events in serum from donors having differing AAT phenotypes. PMNs were incubated on fibronectin while bathed in serum from donors having differing AAT phenotypes, as described in Figure 1; then cells were fixed and examined by incident-light fluorescence microscopy. Images were captured, and quantum proteolytic events were measured using MetaMorph software. Note that the area of quantum proteolytic events associated with PMNs bathed in Pi Z serum was nearly 10 -fold greater than that of cells bathed in serum from Pi M, Pi MS, Pi MZ, and Pi SZ individuals. The events were slightly, but significantly, larger when cells were bathed in serum from heterozygotes for AAT deficiency (Pi MS, Pi $M Z$, and Pi SZ), compared with those in serum from Pi M individuals. Data represent the mean of event sizes among the various donors having the same phenotypes; error bars represent SEM. The number of donors for each AAT phenotype is indicated above each bar. ${ }^{*} P<$ 0.05 vs. Pi M. ${ }^{*} P<0.001$ vs. all other groups. The numbers of events measured in $\mathrm{Pi} \mathrm{M}, \mathrm{Pi} \mathrm{MS}, \mathrm{Pi} \mathrm{MZ}, \mathrm{Pi} \mathrm{SZ}$, and $\mathrm{Pi} \mathrm{Z}$ donors were $612,349,504,245$, and 348 , respectively.

quantum proteolytic events were captured using a Photometrics CH250 chilled CCD imager. MetaMorph software (Universal Imaging, West Chester, Pennsylvania, USA) was used to process the digital images and to measure the sizes of the quantum proteolytic events.

Modeling of quantum proteolytic events. Mathematical modeling of the diffusion of HLE after PMN degranulation was performed using Mathcad for Windows (Mathsoft, Cambridge, Massachusetts, USA). We used a solution to the Fick equations of diffusion for cylindrical geometry from a finite source of known concentration $(2,15)$. This solution defines concentration of HLE $(C)$ as a function of radius $(r)$ and time $(t)$. We took $C_{0}$, the initial concentration of elastase in azurophil granules, to be $5.33 \mathrm{mM}(2) ; D$, the diffusion coefficient for HLE, to be $0.954 \times 10^{-6} \mathrm{~cm}^{2} \mathrm{~s}^{-1}(2,16,17)$; and $a$, the geometric mean radius of the azurophil granule, to be $0.171 \mu \mathrm{m}(2,18)$. We calculated the concentration of HLE at $0.01-\mu \mathrm{m}$ intervals from 0 to $15 \mu \mathrm{m}$ from the center of degranulation, at 1-millisecond time intervals from 1 to 130 milliseconds after degranulation. From the resulting matrix of numerical solutions, we constructed 2-dimensional contour maps of the predicted HLE concentration at various times in the space surrounding a degranulation event, using SigmaPlot (SPSS Science, Chicago, Illinois, USA).
Statistics. The results for unpaired data were compared using Kruskal-Wallis 1-way ANOVA on ranks, and Dunn's pairwise multiple comparisons, using the SigmaStat software package; $P$ values less than 0.05 were considered significant.

\section{Results}

Immunoreactive AAT levels in serum from donors of varying AAT phenotypes. Immunoreactive AAT was quantified in serum from individuals with Pi M, Pi MS, Pi MZ, Pi SZ, and Pi Z AAT phenotypes (Table 1). The values for each phenotype were similar to those reported in other series (5), and none of the values in our study were outside the expected range for any of the phenotypes.

Quantum proteolytic events in serum from donors with varying AATphenotypes. We used an experimental model of PMNs migrating on opsonized fluoresceinated fibronectin (11) while they were bathed in serum, to observe and measure quantum proteolytic events in the presence of serum from donors with varying AAT phenotypes. We have shown that HLE is the predominant proteinase that mediates extracellular proteolytic activity in this model (11).

Cells that were bathed in Pi MZ and Pi SZ serum (Figure 1, b and c) were associated with discrete, rounded proteolytic events that were similar in size and appearance to those observed in the presence of Pi M serum (Figure 1a). However, PMNs that were bathed in Pi $Z$ serum (Figure 1d) generated quantum proteolytic events that were strikingly larger than those that occurred in the presence of serum containing all other AAT phenotypes that were tested.

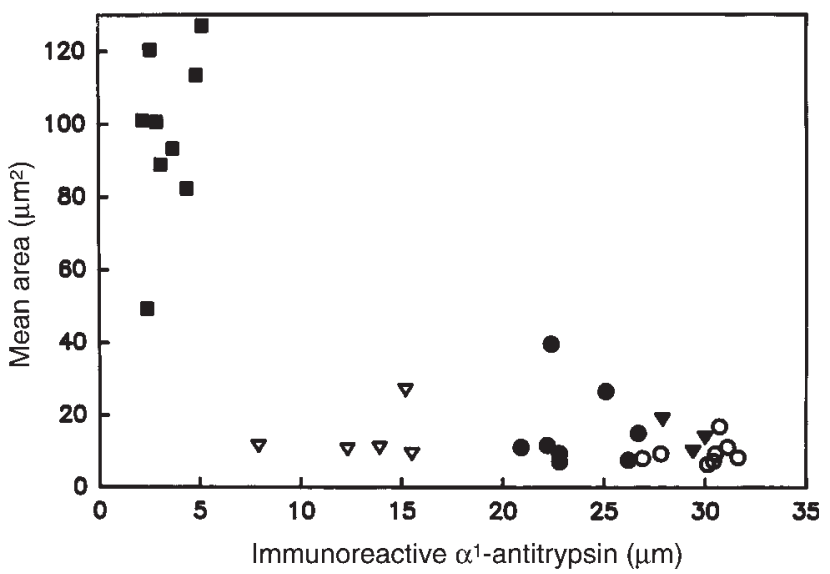

Figure 3

Size of quantum proteolytic events as a function of AAT concentration in serum. AAT concentrations in serum from the donors in Figure 2 (Pi M, open circles; Pi MS, filled triangles; Pi MZ, filled circles; $\mathrm{Pi} \mathrm{SZ}$, open triangles; and $\mathrm{Pi} Z$, filled squares) were measured using an automated fluorescence immunoassay. AAT concentrations (horizontal axis) were plotted against size of the PMN-associated quantum proteolytic events (vertical axis). Note that at AAT concentrations less than approximately $10 \mu \mathrm{M}$, small changes in serum AAT were associated with striking increases in the size of quantum proteolytic events, whereas the sizes of the events changed minimally at higher AAT concentrations. 
Table 1

Immunoreactive AAT in serum from donors having differing AAT phenotypes

\begin{tabular}{|c|c|c|}
\hline AAT phenotype ${ }^{A}$ & No. of donors & $\operatorname{AAT}(\mu \mathrm{M})^{\mathrm{E}}$ \\
\hline Pi M & 8 & $31.7 \pm 1.8$ \\
\hline Pi MS & 3 & $30.8 \pm 1.1$ \\
\hline Pi MZ & 8 & $25.0 \pm 2.2$ \\
\hline PiSZ & 5 & $13.3 \pm 3.0$ \\
\hline Pi Z & 9 & $3.7 \pm 1.2$ \\
\hline
\end{tabular}

${ }^{A}$ AAT phenotype of each donor was determined by isoelectric focusing in polyacrylamide gels (12). ${ }^{B}$ AAT concentrations were determined by automated fluorescence immunoassay (13). ${ }^{C}$ Data are mean \pm SD.

We then measured PMN-associated quantum proteolytic events using quantitative image analysis. Quantum proteolytic events in Pi MS, Pi MZ, and Pi SZ were only slightly, but significantly, larger $(P<0.05)$ than those in Pi M serum. However, the events did not differ significantly in size when the various heterozygote groups were compared. The quantum proteolytic events that were measured in serum from individuals with AAT deficiency (Pi Z) were significantly and strikingly ( 10-fold) larger than those in normal serum, as well as those in all of the heterozygote groups (Figure 2; $P<0.001$ ).

When we assessed the size of the quantum proteolytic events as a function of the AAT concentrations in the experiments containing serum from the various donors, there was a striking inverse and nonlinear relationship between the area of the event and AAT concentration (Figure 3). This relationship was markedly curvilinear with an inflection below $10 \mu \mathrm{M}$, such that relatively small reductions in AAT concentrations occurring at low concentrations result in large increases in the areas of the events, whereas relatively large increases at higher serum AAT concentrations result in small decreases in the areas of the quantum proteolytic events.

To provide assurance that the increased size of quantum proteolytic events associated with PMNs bathed in $\mathrm{Pi} Z$ serum was directly attributable to the lower concentration of AAT in Pi Z serum, we measured quantum proteolytic events associated with cells bathed in serial dilutions of serum from a Pi M individual (initial AAT concentration $41.6 \mu \mathrm{M})$. The relationship between the event sizes and AAT concentration is shown in Figure 4. It was especially noteworthy that the event areas in serum diluted 1:2 (with an AAT concentration that might be observed in a heterozygote for AAT deficiency) were only slightly, but significantly, greater than those occurring in undiluted Pi M serum. Figure 4 also shows that the event areas associated with cells bathed in further-diluted serum equaled those measured in undiluted serum from a Pi $Z$ individual. These results show that reducing the inhibitor concentration in serum by a process of dilution recapitulates the effects of heterozygous and AAT-deficient serum on the sizes of PMN-associated proteolytic events.
Mathematical modeling of single granule events. Our imaging system cannot track the progress of rapid $(<2$ seconds) proteolytic events (11). Because the quantum bursts of proteolysis are completed within this time frame (11), we turned to a mathematical simulation to provide information about the duration of catalytic activity of HLE after degranulation that is not obtainable by direct experimentation. We calculated the theoretical concentration of HLE at 1,500 radii from a site of granule extrusion for various time intervals after degranulation.

Figure 5 is a 2-dimensional illustration of predicted HLE concentrations at 10, 20, 80, and 90 milliseconds after degranulation. Note that at 10 milliseconds (Figure 5a), $30 \mu \mathrm{M}$ HLE (which is approximately the concentration of AAT in normal Pi M serum) is approaching its maximal radius of $1.38 \mu \mathrm{m}$ from the site of degranulation. Within 20 milliseconds (Figure 5b), however, the highest residual HLE concentration has decreased to a value below $30 \mu \mathrm{M}$. Thus, at 20 milliseconds after degranulation, the local HLE concentration no longer would be expected to exceed the normal serum concentration of AAT. Note also in Figure 5b that $15 \mu \mathrm{M}$ HLE (which is approximately the concentration of AAT in Pi SZ serum) is approaching its maximal radius of $1.95 \mu \mathrm{m}$ at 20 milliseconds after degranulation. At 28 milliseconds after degranulation (not shown), the highest residual HLE concentration has decreased below $15 \mu \mathrm{M}$ and thereafter would be expected to be lower than the plasma AAT concentration in Pi SZ subjects. In marked contrast (Figure 5c), a region with $5 \mu \mathrm{M}$ residual HLE concentration (which is

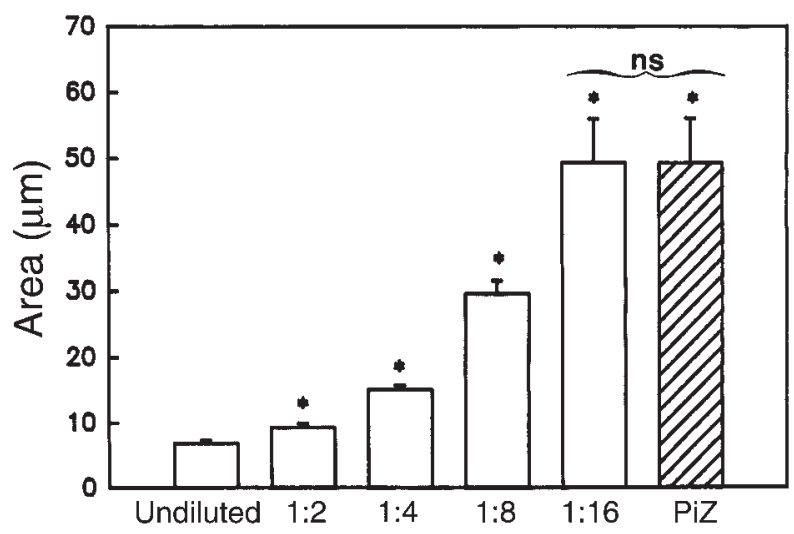

Figure 4

Size of quantum proteolytic events in serial dilutions of Pi M serum. PMNs were incubated on opsonized, fluoresceinated fibronectin while bathed in serum that was either undiluted or diluted in HBSS: Pi M serum, open bars; Pi Z serum, hatched bars. Quantum proteolytic events were imaged and measured as described in Methods. Note the increase in event area when inhibitor concentration is reduced by diluting Pi $\mathrm{M}$ serum. The event areas in Pi M serum diluted 1:16 did not differ from those in undiluted $\mathrm{Pi} Z$ serum $(P=0.50)$. Data are mean \pm SEM. ${ }^{*} P<0.001$ vs. cells bathed in undiluted $P i M$ serum. The numbers of events measured in Pi M serum (undiluted and diluted $1: 2,1: 4,1: 8$, and $1: 16$ ) and undiluted $\mathrm{Pi} Z$ serum were $82,64,32,56,38$, and 18 , respectively. 
approximately the AAT concentration in AAT-deficient [Pi Z] serum) still is found at 80 milliseconds after degranulation. This HLE concentration has dispersed by 90 milliseconds (Figure $5 \mathrm{~d}$ ).

When the matrix of solutions to the diffusion model is examined, an HLE concentration equal to the mean AAT concentration found in the serum of our Pi M subjects $(31.7 \mu \mathrm{M})$ occupies a maximal area of $5.7 \mu \mathrm{m}^{2}$ and disperses by 13 milliseconds (Table 2). In marked contrast, Table 2 also shows that an HLE concentration equal to the mean AAT concentration found in the serum of $\mathrm{Pi} Z$ subjects in this study $(3.7 \mu \mathrm{M})$ occupies a maximal area of $48.8 \mu \mathrm{m}^{2}$, and persists until 110 milliseconds after degranulation. These data predict that in AAT deficiency (phenotype Pi Z), the extracellular area and duration for which the molar ratio of HLE to AAT exceeds 1:1 after a PMN degranulation event (and obligate catalytic activity must exist) are each increased by nearly 9-fold when compared with Pi $M$ (normal) individuals.

Table 2 also shows predicted area and duration of quantum proteolytic events in Pi MS, Pi MZ, and Pi SZ heterozygotes. In the Pi MZ and Pi SZ individuals, there are smaller, but definite, predicted differences in quantum proteolytic events, which are greatest in the Pi SZ individuals (2.4-fold increase in both size and duration when compared with Pi M individuals).

Activation of cells with proinflammatory mediators. To test the reproducibility of our measurements when PMNs are activated by different signals, we assessed the effects of proinflammatory mediators on the size of proteolytic events associated with PMNs bathed in Pi M serum. As expected (19), inactivated PMNs that were adherent to unopsonized fibronectin were not associated with proteolytic events (data not shown). Cells that were adherent to opsonized fibronectin and preactivated with FMLP $\left(10^{-8} \mathrm{M}\right.$ for 30 minutes $)$, with or without priming with LPS (100 ng/mL for 2 minutes), produced quantum proteolytic events with areas of $6.63 \pm(\mathrm{SEM}) 0.27 \mu \mathrm{m}^{2}(n=189)$ and $6.65 \pm 0.25 \mu \mathrm{m}^{2}(n$ $=201)$, respectively $(P=0.985)$. The sizes of these events did not differ from those produced by PMNs that were not activated before their adherence to opsonized fibronectin $\left(\right.$ area $=7.05 \pm 0.32 \mu \mathrm{m}^{2}, n=114 ; P=0.1$ for both comparisons).

\section{Discussion}

To our knowledge, this is the first study to measure the areas of quantum proteolytic events associated with PMNs bathed in a biologic fluid. Diffusion modeling, beginning from first principles (2), also allowed prediction of the very brief duration of catalytic activity in the quantum proteolytic events, which we could not determine from direct observations. Our measurements and models of these events in serum from donors with various AAT phenotypes provide new insights into the mechanisms of lung tissue injury in AAT deficiency.

The quantum proteolytic events that we observed and modeled in serum containing varying AAT concentra-
Table 2

Predicted size and duration of quantum proteolytic events in serum from donors having differing AAT phenotypes

\begin{tabular}{lcc}
\cline { 2 - 2 } AAT phenotype & Predicted area $\left(\mu \mathrm{m}^{2}\right)^{\mathrm{B}, \mathrm{C}}$ & Predicted duration $(\mathrm{ms})^{\mathrm{B}, \mathrm{D}}$ \\
Pi M & 5.7 & 13 \\
Pi MS & 5.9 & 13 \\
Pi MZ & 7.2 & 16 \\
Pi SZ & 13.7 & 31 \\
Pi Z & 48.8 & 110
\end{tabular}

AAAT phenotype of each donor was determined by isoelectric focusing in polyacrylamide gels (12). BPredictions from diffusion modeling, based upon mean serum AAT concentrations of the phenotypic groups described in the text. CPredicted areas are derived from the largest area at any time after degranulation, and are encompassed by an HLE concentration equal to that of mean serum concentration of AAT in the respective phenotypic groups. ${ }^{D}$ Durations for which HLE concentrations exceed mean serum AAT concentrations in the respective phenotypic groups.

tions demonstrated that there was an inverse and strikingly nonlinear relationship between the size of the quantum proteolytic events and the serum AAT concentration. Small changes in serum AAT below $10 \mu \mathrm{M}$ were associated with very large increases in the areas of quantum proteolytic events, whereas large increases in serum AAT concentrations above $10 \mu \mathrm{M}$ had much smaller effects on the areas of these events. In particular, we showed that quantum proteolytic events in the presence of serum from $\mathrm{Pi} \mathrm{Z}$ donors were strikingly abnormal (9- to 10-fold greater both in area and duration when compared with those in Pi M serum). Interestingly, when PMNs were bathed in serum from AAT heterozygotes containing intermediate concentrations of AAT, the quantum proteolytic events were only slightly, but significantly, increased in size and duration when compared with those in Pi M serum.

Our data provide a new construct for understanding tissue injury in AAT deficiency. They suggest that in Pi $\mathrm{Z}$ donors, low plasma concentrations of AAT alone lead to abnormal quantum proteolytic events, a result not predicted by traditional kinetic models of enzymeinhibitor interactions. Thus, after PMN degranulation in $\mathrm{Pi} \mathrm{Z}$ individuals, quantum proteolytic events can be expected to occupy a much larger extracellular volume and to be much more persistent than in normal individuals. These data indicate that a potentially important mechanism by which extracellular proteins are at risk in Pi $\mathrm{Z}$ individuals is through defective AAT-mediated confinement of HLE catalytic activity. $\alpha_{2}$ Macroglobulin (the other major circulating inhibitor of HLE) is present in substantially lower concentrations in plasma when compared with plasma AAT levels, even in individuals with AAT deficiency. $\alpha_{2}$-Macroglobulin exerts minimal effects on the sizes of the proteolytic events that we studied, because the sizes of the events were very similar to those observed in buffers containing various concentrations of AAT alone (11).

The above construct does not conflict with earlier studies of HLE-AAT interactions in AAT deficiency (9, 20). Although inhibitor concentration has a dominant 

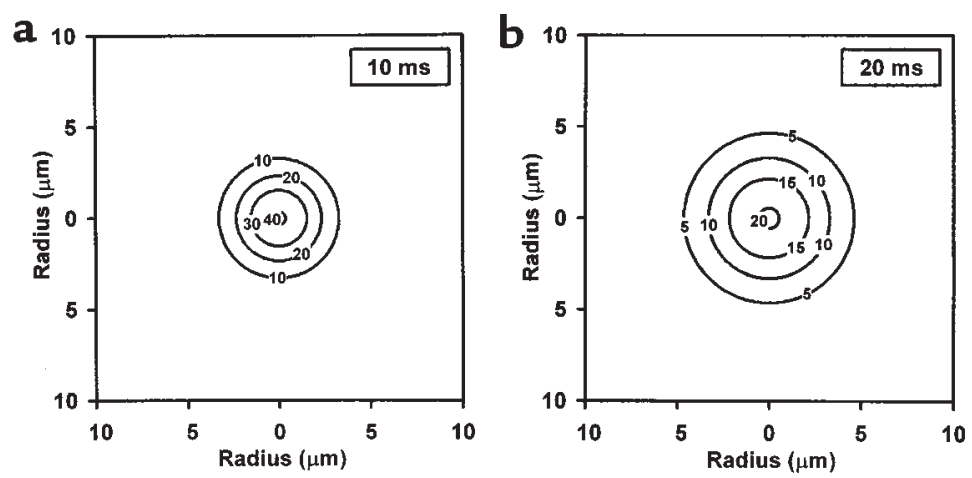

Figure 5

Predicted HLE concentrations at various distances from the site of degranulation, as a function of time. The center of each graph is the site of degranulation, with distance increasing away from this site. The horizontal and vertical axes extend to $10 \mu \mathrm{m}$ from the site of granule release. The circles in the graphs each represent discrete concentrations of $\operatorname{HLE}(\mu \mathrm{M})$ at the indicated times (10-90 milliseconds) after degranulation. Data are derived from diffusion modeling, as described in the text. In a, note that HLE concentrations of $30 \mu \mathrm{M}$ and greater are present inside at approximately $1 \mu \mathrm{m}$ at 10 milliseconds after granule extrusion, but have dispersed by 20 milliseconds (b).
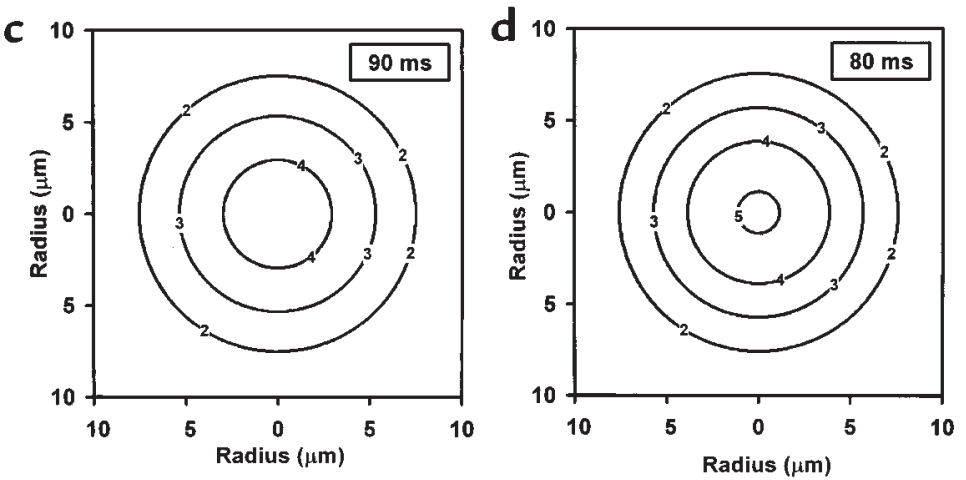
In marked contrast, HLE concentrations of $5 \mu \mathrm{M}$ and greater persist at 80 milliseconds (c), but have dispersed by 90 milliseconds $(\mathbf{d})$.

effect on the size and duration of quantum proteolytic events, altered inhibition kinetics may also contribute to HLE-mediated tissue injury in AAT deficiency.

The discovery of AAT deficiency and its association with an increased risk of development of pulmonary emphysema $(1,21)$ was a critically important event in the evolution of the "proteinase-antiproteinase hypothesis" of pulmonary emphysema. Relevant observations are as follows: (a) HLE is the key biologic target of AAT (22); (b) injury to lung parenchymal elastin is a pivotal event in the pathogenesis of pulmonary emphysema (23); and (c) HLE can degrade elastin and cause emphysema in experimental animals $(24,25)$. According to this hypothesis, defective inhibition of HLE has been presumed to be a feature of AAT deficiency. Indeed, patients with AAT deficiency have increased plasma concentrations of fibrinopeptides that are uniquely produced by the catalytic activity of HLE (26). The present work provides a new mechanistic explanation for that observation and provides further support for the applicability of the proteinase-antiproteinase hypothesis to lung injury in pulmonary emphysema.

Some studies $(7,27-29)$, but not all (30-32), have suggested a slightly increased risk of lung disease in heterozygotes for AAT deficiency. It is likely that, in the presence of cigarette smoking and familial or other additive risk factors, some heterozygotes do experience an increased risk of developing pulmonary emphysema $(6,33)$. At a mechanistic level, it is noteworthy that a study by Weitz et al. (26) showed significantly increased levels of HLE-specific fibrinopeptides among heterozygotes for AAT deficiency. The present work, which showed that quantum proteolytic events were slightly, but significantly, larger and more prolonged in serum from patients who were heterozygotes for AAT deficiency, provides conceptual support for the possibility that, in certain clinical settings, the heterozygous state may be associated with a somewhat increased risk of injury to the lung and other tissues.

Any mechanism that causes an increase in uninhibited HLE activity, including the overwhelmingly high transient local HLE concentrations described here, has the potential to injure extracellular proteins within the immediate environment of activated inflammatory cells. In addition, these obligate bursts of HLE activity might promote tissue inflammation, because HLE has proinflammatory activities (34-36). In this respect, it is noteworthy that AAT deficiency is also associated with inflammation and tissue injury in vascular and adipose tissue, as well as the lung. For example, patients with AAT deficiency and heterozygotes for the disease (37-40) have a markedly increased risk of development of necrotizing vasculitis. Although uncommon, necrotizing panniculitis and membranoproliferative glomerulonephritis have also been associated with AAT deficiency (41-43).

In the present analyses of the risks of tissue injury associated with AAT deficiency, we have based our discussion on plasma concentrations of AAT, whereas many of the potentially injurious processes of interest occur in the interstitial space. AAT concentrations in pulmonary epithelial lining fluid have been estimated based upon data from bronchoalveolar lavage (44), and are approximately 10 -fold lower than those in plasma (3.4 and $32.8 \mu \mathrm{M}$, respectively). Although there have been no direct studies of AAT concentrations in interstitial fluid, the available evidence suggests that plas- 
ma and interstitial fluid AAT concentrations are similar, even when inflammation and structural injury are absent. Ultrastructural studies (45) have shown that pulmonary epithelial barriers are much tighter than endothelial barriers, and these observations are in good agreement with physiological studies in animals, demonstrating that $92-98 \%$ of the resistance to plasma protein flux across the alveolar-capillary membrane lies in the epithelial barrier (46). Thus, pulmonary interstitial AAT concentrations can be expected to be much closer to those in plasma than to those in epithelial lining fluid. It is noteworthy that AAT is similar in size to albumin (52 and $67 \mathrm{kDa}$, respectively), and that albumin has been measured in pulmonary lymph (which drains the interstitial space) in conscious sheep (47). The ratio of pulmonary lymph to plasma albumin in sheep ranges from 0.78 to $0.87(47,48)$. Given the smaller molecular radius of AAT, its lymph/plasma ratio would be expected to be slightly higher than that for albumin.

At sites of inflammation in the lung and other organs, endothelial permeability increases further (47, 48 ), and it is likely that at sites of potential tissue injury interstitial AAT concentrations closely resemble those in plasma. Finally, it must be noted that AAT is an acute-phase reactant in normal individuals, whereas AAT-deficient individuals are capable of increasing plasma AAT concentrations only minimally. Thus, the discrepancy in plasma (and interstitial) AAT concentrations between normal and AAT-deficient individuals would be expected to increase during acute stress and/or inflammation. This suggests that during illness or injury, the differences in the size and duration of the quantum proteolytic events between $\mathrm{Pi} \mathrm{M}$ and $\mathrm{Pi} \mathrm{Z}$ individuals may be even greater than those observed during baseline states.

Augmentation therapy with purified human AAT has been approved for use in patients with documented AAT deficiency (plasma AAT $<11 \mu \mathrm{M}$ ) and airflow obstruction who have stopped smoking. Although a randomized study of augmentation therapy has yet to be done, recent studies suggest the possibility that augmenting the plasma AAT concentration to levels above $11 \mu \mathrm{M}$ may slow the rate of decline of lung function $(49,50)$ in AAT-deficient individuals. Our results provide unique insights into the mechanisms by which quantitatively relatively minor augmentation of plasma AAT concentrations may provide therapeutic benefit. Although our observations and predictions do not allow assignment of a specific target value for AAT augmentation, they are in reasonable agreement with the goal of maintaining a plasma level in excess of a clinically estimated $11-\mu \mathrm{M}$ threshold value. Given the nonlinear relationship of quantum event sizes to extracellular inhibitor concentrations, this value is approximately the AAT level below which relatively small increases in plasma AAT concentrations result in large increases in quantum proteolytic event size and duration. However, the present data (e.g., the predic- tions in Table 2) also suggest the possibility of additional benefit from more intense augmentation, with the goal of achieving plasma levels higher than those found in Pi SZ heterozygotes. Finally, our results predict that therapeutic strategies that are designed to decrease intracellular HLE activity within inflammatory cells by inhibiting HLE in situ within azurophil granules (and thereby reducing initial concentrations of active HLE at sites of degranulation) are an alternative and potentially very effective strategy to reduce tissue injury in AAT deficiency.

In summary, we have demonstrated and modeled scenarios that lead to striking dysregulation of quantum proteolytic events that occur near the surface of activated PMNs. These results provide a new construct for understanding tissue injury in AAT deficiency. They also have implications for preventive therapies against injury to the lungs and other tissues in patients who have AAT deficiency.

\section{Acknowledgments}

This work was supported by United States Public Health Service grant HL-46440, The American Lung Association, The Francis Families Foundation, and The Council for Tobacco Research, USA., Inc. C.A. Owen is a Parker B. Francis Fellow in Pulmonary Research.

1. Laurell, C.-B., and Eriksson, S. 1963. The electrophoretic alpha-1-globulin pattern of serum in alpha-1-antitrypsin deficiency. Scand. J. Clin. Lab. Invest. 15:132-140.

2. Liou, T.G., and Campbell, E.J. 1995. Non-isotropic enzyme-inhibitor interactions: a novel non-oxidative mechanism for quantum proteolysis by human neutrophils. Biochemistry. 34:16171-16177.

3. Owen, C.A., et al. 1994. A discrete subpopulation of human monocytes expresses a neutrophil-like pro-inflammatory (P) phenotype. Am. J. Physiol. 267:L775-L785.

4. Janoff, A. 1985. Elastases and emphysema. Current assessment of the protease-antiprotease hypothesis. Am. Rev. Respir. Dis. 132:417-433.

5. Brantly, M.L., et al. 1991 . Use of a highly purified $\alpha_{1}$-antitrypsin standard to establish ranges for the common normal and deficient $\alpha_{1}$-antitrypsin phenotypes. Chest. 100:703-708.

6. Hutchison, D.C.S. 1988. Natural history of alpha-1-protease inhibitor deficiency. Am. J. Med. 84(Suppl. 6A):3-12.

7. Turino, G.M., et al. 1996. Clinical features of individuals with $\mathrm{Pi} * \mathrm{SZ}$ phenotype of alpha 1-antitrypsin deficiency. Alpha 1-Antitrypsin Deficiency Registry Study Group. Am. J. Respir. Crit. Care Med. 154:1718-1725.

8. Beatty, K., Matheson, N., and Travis, J. 1984. Kinetic and chemical evidence for the inability of oxidized alpha $_{1}$-proteinase inhibitor to protect lung elastin from elastolytic degradation. Hoppe Seylers Z. Physiol. Chem. 365:731-736.

9. Ogushi, F., Fells, G.A., Hubbard, R.C., Straus, S.D., and Crystal, R.G. 1987. $Z$-type alpha ${ }_{1}$-antitrypsin is less competent than M1-type alpha ${ }_{1}$-antitrypsin as an inhibitor of neutrophil elastase. J. Clin. Invest. 80:1366-1374.

10. Llewellyn-Jones, C.G., Lomas, D.A., and Stockley, R.A. 1994. Potential role of recombinant secretory leucoprotease inhibitor in the prevention of neutrophil mediated matrix degradation. Thorax. 49:567-572.

11. Liou, T.G., and Campbell, E.J. 1996. Quantum proteolysis resulting from release of single granules by neutrophils: a novel, non-oxidative mechanism of extracellular proteolytic activity. J. Immunol. 157:2624-2631.

12. Pierce, J.A., and Eradio, B.G. 1979. Improved identification of antitrypsin phenotypes through isoelectric focusing with dithioerythritol. J. Lab. Clin. Med. 94:826-831.

13. Silverman, E.K., et al. 1989. Alpha 1-antitrypsin deficiency: high prevalence in the St. Louis area determined by direct population screening. Am. Rev. Respir. Dis. 140:961-966.

14. Boyum, A. 1963. Isolation of mononuclear cells and granulocytes from human blood: isolation of mononuclear cells by one centrifugation and of granulocytes by combining centrifugation and sedimentation at $1 \mathrm{~g}$. Scand. J. Clin. Lab. Invest. Suppl. 97:77-89.

15. Crank, J. 1975. The mathematics of diffusion. Oxford University Press. New York, NY. 28-43. 
16. Baugh, R.J., and Travis, J. 1976. Human leukocyte granule elastase: rapid isolation and characterization. Biochemistry . 15:836-841.

17. Ohlsson, K., and Olsson, I. 1974. The neutral proteases of human granulocytes: isolation and partial characterization of granulocyte elastase. Eur. J. Biochem. 42:519-527

18. Damiano, V.V., Kucich, U., Murer, E., Laudenslager, N., and Weinbaum, G. 1988. Ultrastructural quantitation of peroxidase- and elastase-containing granules in human neutrophils. Am. J. Pathol. 131:235-245.

19. Campbell, E.J., and Campbell, M.A. 1988. Pericellular proteolysis by neutrophils in the presence of proteinase inhibitors: effects of substrate opsonization. J. Cell Biol. 106:667-676.

20. Llewellyn-Jones, C.G., Lomas, D.A., Carrell, R.W., and Stockley, R.A. 1994. The effect of the $\mathrm{Z}$ mutation on the ability of $\alpha_{1}$-antitrypsin to prevent neutrophil mediated tissue damage. Biochim. Biophys. Acta. 1227:155-160.

21. Eriksson, S. 1964. Pulmonary emphysema and alpha ${ }_{1}$-antitrypsin deficiency. Acta Med. Scand. 175:197-205.

22. Beatty, K., Bieth, J., and Travis, J. 1980. Kinetics of association of serine proteinases with native and oxidized alpha-1-proteinase inhibitor and alpha-1-antichymotrypsin. J. Biol. Chem. 255:3931-3934.

23. Shapiro, S.D. 1995. The pathogenesis of emphysema: the elastase:antielastase hypothesis 30 years later. Proc. Assoc. Am. Physicians. 107:346-352.

24. Senior, R.M., et al. 1977. The induction of pulmonary emphysema with human leukocyte elastase. Am. Rev. Respir. Dis. 116:469-475.

25. Janoff, A., et al. 1977. Experimental emphysema induced with purified human neutrophil elastase. Tissue localization of the instilled protease. Am. Rev. Respir. Dis. 115:461-478.

26. Weitz, J.I., Silverman, E.K., Thong, B., and Campbell, E.J. 1992. Plasma levels of elastase-specific fibrinopeptides correlate with proteinase inhibitor phenotype. Evidence for increased elastase activity in subjects with homozygous and heterozygous deficiency of $\alpha_{1}$-proteinase inhibitor. J. Clin. Invest. 89:766-773.

27. Larsson, C., Eriksson, S., and Dirksen, H. 1977. Smoking and intermediate alpha 1-antitrypsin deficiency and lung function in middle-aged men. Br. Med. J. 2:922-925.

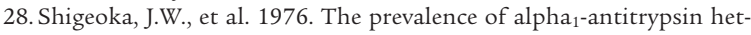
erozygotes (Pi MZ) in patients with obstructive pulmonary disease. Am. Rev. Respir. Dis. 114:1077-1084.

29. Fagerhol, M.K., and Hauge, H.E. 1969. Serum Pi types in patients with pulmonary diseases. Acta Allergol. 24:107-114.

30. Cole, et al. 1976. Relation of alpha-1-antitrypsin phenotype to the performance of pulmonary function tests and to the prevalence of respiratory illness in a working population. Thorax. 31:149-157.

31. Hutchison, D.C.S., Tobin, M.J., and Cook, P.J.L. 1983. Alpha ${ }_{1}$-antitrypsin deficiency: clinical and physiological features in heterozygotes of type Pi SZ. A survey by the British Thoracic Association. Br. J. Dis. Chest. 77:28-34

32. Buist, A.S., Sexton, G.J., Azzam, A.-M.H., and Adams, B.E. 1979. Pul-

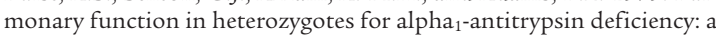
case-control study. Am. Rev. Respir. Dis. 120:759-766.

33. Mittman, C. 1978. The PiMZ phenotype: is it a significant risk factor for the development of chronic obstructive lung disease? Am. Rev. Respir. Dis. 118:649-652

34. Hubbard, R.C., et al. 1991. Neutrophil accumulation in the lung in $\alpha 1$ antitrypsin deficiency. Spontaneous release of leukotriene $\mathrm{B}_{4}$ by alveolar macrophages. J. Clin. Invest. 88:891-897.

35. Bedard, M., et al. 1993. Release of interleukin-8, interleukin-6, and colony-stimulating factors by upper airway epithelial cells: implications for cystic fibrosis. Am. J. Respir. Cell Mol. Biol. 9:455-462.

36. Nakamura, H., Yoshimura, K., McElvaney, N.G., and Crystal, R.G. 1992. Neutrophil elastase in respiratory epithelial lining fluid of individuals with cystic fibrosis induces interleukin- 8 gene expression in a human bronchial epithelial cell line. J. Clin. Invest. 89:1478-1484.

37. Elzouki, A.N., Segelmark, M., Wieslander, J., and Eriksson, S. 1994. Strong link between the alpha 1-antitrypsin Pi Z allele and Wegener's granulomatosis. J. Intern. Med. 236:543-548.

38. Mazodier, P., Elzouki, A.N., Segelmark, M., and Eriksson, S. 1996. Systemic necrotizing vasculitides in severe alpha ${ }_{1}$-antitrypsin deficiency. QJM. 89:599-611.

39. Callea, F., et al. 1997. Alpha 1-antitrypsin (AAT) deficiency and ANCApositive systemic vasculitis: genetic and clinical implications. Eur.J. Clin. Invest. 27:696-702.

40. O’Donoghue, D.J., Guickian, M., Blundell, G., and Winney, R.J. 1993. Alpha-1-proteinase inhibitor and pulmonary haemorrhage in systemic vasculitis. Adv. Exp. Med. Biol. 336:331-335.

41. Su, W.P., Smith, K.C., Pittelkow, M.R., and Winkelmann, R.K. 1987. Alpha 1-antitrypsin deficiency panniculitis: a histopathologic and immunopathologic study of four cases. Am. J. Dermatopathol. 9:483-490.

42. Bleumink, E., and Klokke, H.A. 1984. Protease-inhibitor deficiencies in a patient with Weber-Christian panniculitis. Arch. Dermatol. 120:936-940.

43. Lewis, M., et al. 1985. Severe deficiency of alpha 1-antitrypsin associated with cutaneous vasculitis, rapidly progressive glomerulonephritis, and colitis. Am. J. Med. 79:489-494.

44. Wewers, M.D., et al. 1987. Replacement therapy for alpha -antitrypsin $^{-}$ deficiency associated with emphysema. N. Engl. J. Med. 316:1055-1062.

45. Schneeberger, E.E. 1979. Barrier function of intercellular junctions in adult and fetal lungs. In Pulmonary edema. A.P. Fishman and E.M. Renkin, editors. American Physiological Society. Bethesda, MD. 21-37.

46. Gorin, A.B., and Stewart, P.A. 1979. Differential permeability of endothelial and epithelial barriers to albumin flux. J. Appl. Physiol. 47:1315-1324.

47. Brigham, K.L., and Owen, P.J. 1975. Increased sheep lung vascular permeability caused by histamine. Circ. Res. 37:647-657.

48. Brigham, K.L., and Owen, P.J. 1975. Mechanism of the serotonin effect on lung transvascular fluid and protein movement in awake sheep. Circ. Res. 36:761-770.

49. The Alpha-1-Antitrypsin Deficiency Registry Study Group. 1998. Survival and $\mathrm{FEV}_{1}$ decline in individuals with severe deficiency of $\alpha_{1}$-antitrypsin. Am. J. Respir. Crit. Care Med. 158:49-59.

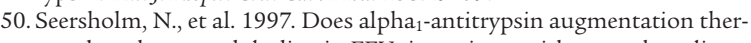
apy slow the annual decline in $\mathrm{FEV}_{1}$ in patients with severe hereditary alpha $_{1}$-antitrypsin deficiency? Eur. Respir. J. 10:2260-2263. 